\title{
Implantation Failure, Etiology, Diagnosis and Treatment
}

\author{
${ }^{1}$ Yoel Shufaro, ${ }^{2}$ Joseph G Schenker \\ ${ }^{1}$ Department of Obstetrics and Gynecology, Hebrew University, Hadassah Medical Center, Jerusalem, Israel \\ ${ }^{2}$ Professor and Chairman (Emeritus), Department of Obstetrics and Gynecology, Hebrew University, Hadassah Medical Center \\ Jerusalem, Israel
}

Correspondence: Yoel Shufaro, Department of Obstetrics and Gynecology, Hebrew University, Hadassah Medical Center POB 12000, Jerusalem-91120, Israel, Phone: +972 2 6777111, Fax: +972 2 6433337, e-mail: yoelsh@ @ekmd.huji.ac.il.

\section{ABSTRACT}

Embryonic implantation is a complex interaction between the embryo and the endometrium. Despite great investigative effort this process is still obscure. Contrary to the great advancement in patient care, follicular recruitment, oocyte quality and aspiration, embryo quality, culture and cryopreservation, our understanding of the implantation process did not enhance as much, and the tools to intervene within this process are limited. The implantation of the transferred embryos still remains the major limiting factor in IVF. Here we will review the current literature on the maternal (uterine, hematologic, immunologic and others) and embryonic factors that are associated with repeated implantation failure (RIF) and describe the various therapeutic approaches to cope with them. In addition, we will present our conclusive recommendations on how to investigate and manage RIF based on the literature and our own experience.

Keywords: In vitro fertilization, Implantation, Repeated implantation failure.

\section{INTRODUCTION}

Repeated implantation failure (RIF) is defined as the failure to achieve a pregnancy following repeated IVF cycles. Though no formal definition exists, it is accepted that 2 to 6 IVF cycles, in which at least 10 high-grade embryos were transferred to the uterus is defined as RIF. ${ }^{1}$ However in most currently operating IVF programs, three unsuccessful ART cycles in which 1 to 2 reasonably good embryos were transferred will attract a special investigative attention. $^{2}$

The process of implantation depends on the communication between the embryo and the endometrium, which produces numerous factors and signals required for successful implantation and pregnancy outcome after IVF. Despite great investigative effort, this process largely remained an enigmatous 'black box'. Patient care, follicular recruitment, oocyte quality and aspiration, embryo quality culture, and cryopreservation have greatly improved since the emergence of IVF more than three decades ago. Stimulation protocols, embryo culture and transfer techniques have been optimized. However, our understanding of the implantation process did not enhance at the same rate, and the tools to intervene within it are extremely limited. Despite a significant increase in IVF success rates up to more than double the spontaneous fecundity of young fertile couples, the implantation of the transferred embryos still remains the major success limiting factor. Although the investigation

Date of Received: 02-11-10

Date of Acceptance: 14-12-10

Date of Publication: Jan. 2011 for a RIF cause is sometimes fruitful, the vast majority of the cases remain obscure or 'idiopathic'. Here, we will describe the maternal (uterine, hematologic, immunologic and others) and embryonic factors which are associated with implantation failure and describe the various therapeutic approaches to cope with them.

\section{MATERNAL FACTORS}

The maternal factors contributing to decreased receptivity are gross uterine anomalies, such as septa, submucous fibroids, endometrial scarring, resulting in thin estrogen unresponsive endometrium with or without adhesions, altered expression of adhesive molecules, states of hypercoagulability and immunological factors.

\section{Uterine Anatomical Anomalies}

Hysteroscopically visible uterine anomalies can be diagnosed in up to a quarter of the patients with a normally appearing cavity in their initial hysterosalpingogram or hysteroscopy. ${ }^{3}$ The contribution of such findings to implantation failure is variable. The impact of lesions minimally, or not distorting the uterine cavity on implantation, remains controversial. However, the surgical correction of gross intracavitary anomalies, such as protruding submucous fibroids, adhesion or long septa was found to be beneficial. ${ }^{3}$ The postsurgical pregnancy rates were higher than initially observed in the same patients, ${ }^{4}$ but no appropriate prospective controlled studies have been performed. It is therefore appropriate, though not entirely evidence based, to re-evaluate the uterine cavity once the diagnosis of RIF is established, and to surgically correct any significant anomaly found. 


\section{Thin Endometrium}

The evidence regarding the importance of endometrial thickness, as measured by ultrasonographic examination, to implantation is equivocal. While some authors have shown a strong association between this parameter to implantation, ${ }^{5-12}$ others have failed to show such a relationship. ${ }^{13-18}$ In some studies, the endometrial thickness was related to the IVF outcome, but only in correlation with other parameters. ${ }^{19,20}$ Different minimal endometrial thickness thresholds were suggested as essential for successful implantation. In most published studies, no pregnancy was achieved when the thickness of the preovulatory endometrium was $<6 \mathrm{~mm}^{21}$ Nevertheless, Sundstrom et $\mathrm{al}^{22}$ have reported a successful outcome of an IVF cycle in a patient with an endometrial thickness of no more than $4 \mathrm{~mm}$.

Several therapeutic approaches have been suggested to overcome the problem of thin endometrium. Low-dose aspirin, ${ }^{23}$ vaginal sildenafil ${ }^{24}$ in addition to stimulation with high-dose oral and vaginal estrogens. ${ }^{25}$ The purpose of these strategies is to increase the global and implantation site endometrial blood flow. Low-dose aspirin was found to have no effect in the general IVF patient population, ${ }^{26}$ but none of these approaches was adequately studied in the IVF patients with RIF. We have published the reproductive results of 99 IVF cycles of a patient cohort with thin unresponsive endometrium who had RIFs. Even if some improvement was achieved employing one of these strategies, the reproductive outcome was still very poor. The pregnancy rate was low, the miscarriage rate was high, and the live birth rate was close to null. ${ }^{27}$ Therefore, it is our belief that this problem has no effective treatment and other solutions like surrogacy or adoption should be sought.

Stimulation of the endometrium by local injury using an endometrial biopsy catheter was reported to be beneficial to patients with normal endometrial thickness who had otherwise unexplained RIF. ${ }^{28,29}$ However in the published studies, the good results of the poststimulation cycles were compared to the results achieved in the same patients before. This methodology is suboptimal. The effectiveness of endometrial stimulation in patients with otherwise unexplained RIF or with thin unresponsive endometrium is to be determined the performance of prospective randomized controlled trials. Another benefit of the endometrial biopsy is the availability of tissue for histological diagnosis. Significant subclinical conditions, such as chronic endometritis can be diagnosed and eventually treated. Another similar approach to RIF (with or without thin endometrium) is to remove the functional endometrium entirely by performing a formal dilatation and curettage, followed by estrogen therapy for the purpose of achieving growth and regeneration of a better endometrium. The benefit of this procedure is not supported by evidence at all, and its performance might even be detrimental. ${ }^{27}$

\section{PELVIC FACTORS}

Patients with hydrosalpinges have lower implantation rates presumably because of the detrimental effect the hydrosalpinx fluid has on the endometrium and possibly on the embryo as well. ${ }^{30,31}$ A systematic review of three RCTs showes that tubal surgery, such as laparoscopic salpingectomy significantly increased live birth rate and pregnancy rate in women with hydrosalpinges before IVF when compared with no treatment. ${ }^{32}$ It is therefore the recommendation of both the American Society for Reproductive Medicine (ASRM, USA) and the National Health Service (NHS, UK), to surgically remove fluid filled distally occluded tubes prior to any IVF treatment. ${ }^{33}$

\section{Altered Expression of Adhesive Molecules}

Local dysregulation of cytokine expression was related to RIF. Elevated endometrial NK cells, dysregulation of interleukins (IL) $)^{12,15}$ and high IL-1 $\beta$ and low interferon- $\gamma$ and IL-10 (Inagaki et al, 2003) were all found in the endometriae of patients suffering from RIF. ${ }^{34,35}$ High levels of aromatase p450 transcription and alterations in pinopode expression have been associated with RIF as well. ${ }^{36,37}$ Although research in this direction is most relevant to resolving the unexplained cases of RIF, no essay or therapeutic strategy of clinical significance based on these studies exists up to date.

\section{States of Hypercoagulability}

The role of inherited and acquired hypercoagulable states (thrombophilia) in RIF is presumed to be in a mechanism similar to recurrent miscarriages. Antiphospholipid and other autoantibodies were associated with RIF has been shown in some early studies, but later large prospective studies performed in the late 90's failed to reveal an association between antiphoshpholipid antibodies and RIF. ${ }^{38,39}$ Part of this ambiguity is caused by the use of different assays to determine the presence of the antibodies tested in different places and times. However, more recent studies, in which up to date examinations including genetic tests for hereditary thrombophilias were used, had associated both inherited and acquired thrombophilias with RIF and poor IVF outcome. In some studies, inherited and acquired thrombophilias were found to be more abundant among the patients with RIF, especially in those defined as having "unexplained infertility" when compared to control populations; the general population and succeeding IVF patients. ${ }^{40,41}$ Moreover, the effectiveness of low molecular weight heparin in increasing the implantation, pregnancy, and live birth rates was proven in some small prospective randomized controlled trials. ${ }^{42}$ On the other hand an equal amount of studies ruling out such a connection or the therapeutic effectiveness of heparin also exists. ${ }^{43-45}$ Thus, screening for thrombophilia in RIF is still controversial, but is performed routinely by many practitioners. ${ }^{1}$ It is our opinion that thrombophilia screening should be a part of the evaluation of RIF. It is our personal impression that once thrombophilia is diagnosed and prophylactic low dose LMW heparin is administered, the ART success rate increase. In addition, heparin prophylaxis is also important for patient safety during the hyper-estrogenic state created by controlled ovarian stimulation $(\mathrm{COH})$ and pregnancy. 


\section{Immunological Factors}

The association between RIF and immune dysregulation is the most difficult to establish and few studies have actually shown that this association is possible. Carp et al suggested that couples sharing HLA alleles are at high risk of RIF and recurrent very early preclinical pregnancy losses. ${ }^{46}$ In this study, performed in HLA similar couples, antipaternal complement-dependent antibodies were assayed and mixed culture with the male partners' lymphocytes was performed. The (female) patients were immunized with the male partners' lymphocytes if both humoral and cellular antipaternal assays were negative. The study population included IVF patients with recurrent biochemical pregnancies, RIF and early miscarriages. Other IVF cycles were attempted after antipaternal immune response was produced, and resulted in a significantly higher than expected number of viable ongoing pregnancies in all patient groups. ${ }^{46}$ However, this preliminary report has never been confirmed and alloimmunization with another person's lymphocytes might be risky and detrimental. Elram et al have also established an association between RIF and immune dysfunction. ${ }^{47}$ In this study, couples with at least seven unsuccessful ETs that were found to share at least three HLA loci, and had a negative cross-match test were included. The therapeutic intervention was the administration of nonspecific intravenous immunoglobulins (IVIG); $30 \mathrm{gm}$ before oocyte retrieval, and a second dose as soon as a fetal heart beat was identified. The 10 couples that participated in this study had undergone a total of 98 prior IVF cycles without any implantations occurring. Following a total of 18 IVIG courses, seven women conceived, resulting in six deliveries and one second trimester miscarriage. These results suggest that couples high order RIF, HLA similarity and maternal tolerance to paternal antigens have an immunological basis to their problem and might benefit from an immunomodulatory treatment, such as IVIG. Unlike partner alloimmunization, IVIG therapy is nonhazardous in most cases. The commercially available IVIG preparations originating from pooled blood donations, but are subject to strict preparation and safety regulations assuring they are pathogen free, safe, and of high quality. Due to the paucity of studies establishing an immune etiology to RIF and the high cost of IVIG therapy, we believe that the immune investigation for a RIF cause should be performed last, only in couples with high order RIF after other causes have been ruled out or treated. The tests to be performed are determination of HLA loci and cross-match with the male partner's lymphocytes. In those patients meeting the criteria specified by Carp et $\mathrm{al}^{46}$ or Elram et al, ${ }^{47}$ IVIG therapy is safe and potentially beneficial and should therefore be considered. Partner alloimmunization is not as safe as IVIG therapy and offers no additional advantages.

\section{EMBRYONIC FACTORS}

Despite the significant advancement in human extracorporeal embryo culture, the existing knowledge and tools to investigate and treat RIF due to embryonic causes is limited. The recognized embryonic factors to RIF are genetic abnormalities, suboptimal growth in culture and zona hardening.

\section{Genetic Abnormalities}

Chromosomal abnormalities are not infrequent in human embryos cultured in vitro and such embryos have a reduced implantation potential. The percentage of embryonic aneuploidy was found to be higher in RIF cases than in controls. ${ }^{48,49}$ The disruption of chromosome replication and segregation in a greater than anticipated fraction of the cultured early human embryos might be a common cause for RIF. In most cases the parental karyotypes are normal and the embryonic chromosomal aberrations found are incidental or secondary to disturbed gametogenesis. In a minority of cases, a parental balanced translocation is the cause for the generation of aneuploid gametes and embryos. An increased incidence of sperm chromosomal abnormalities was reported in patients with a normal (systemic) karyotype and RIF. ${ }^{50}$ An increased frequency of female (systemic) chromosomal translocations, mosaics, inversions and deletions were observed in young women with high-order RIF. ${ }^{51,52}$

Preimplantation genetic screening (PGS) is the performance of FISH on biopsied blastomeres with probes for the centromeres of the 3 to 8 chromosomes responsible most frequently for aneuploidies. Thus by selecting only the chromosomally normal embryos for transfer, PGS was initially presumed to significantly increase the implantation rates. ${ }^{48,53,54}$ However when a larger prospective study was performed, the use of PGS did not increase but instead significantly reduced the rates of ongoing pregnancies and live births after IVF. ${ }^{55}$ Although the patients enrolled in this study were of advanced age and not necessarily defined as having RIF, the results are relevant to patients with RIF as well as screening for aneploidy does not increase the take-home baby rate.

Parental karyotype determination should be a part of the RIF investigation, especially if a history of miscarriages exists. If a parental translocation or other anomaly is discovered, than preimplantation genetic diagnosis (PGD) is warranted like any other inherited condition. On the other hand, if the parental karyotype is normal, the performance of genetic screening is of no benefit.

\section{Embryo Culture and Transfer}

Presently a large variety of high-quality standard commercially available IVF media for different purposes exists. In some cases, patient specific culture conditions are required for optimal embryonic development. In some RIF cases it might be beneficial to empirically alter the culture media and conditions used when in vitro embryo culture is suboptimal. The evolving proteomic and metabolomic methods assisting with the selection of the embryo with the best implantation potential are not designed for RIF cases, but might prove to be of some benefit in these cases too.

Coculture of embryos with homologous endometrial cells was suggested to improve culture conditions due to the secretion 
of embryotrophic factors, such as nutrients, growth factors and cytokines, and neutralization of harmful substances. ${ }^{56,57}$ Using this method, an impressive pregnancy rate was reported in a large patient group with RIF. ${ }^{58}$ However, most IVF units are not equipped and do not have facilities and personnel required for routine performance of coculture.

Embryo culture (and eventual transfer) to the blastocyst stage harbors several benefits. The blastocyst is placed in the endometrial cavity 5 to 6 days after fertilization, as in natural conception. Culturing the embryos to the blastocyst stage examines the activation of the entire embryonic genome and biologically selects in vitro the embryos with the highest implantation potential. However, in vitro embryo loss is inherent to blastocyst culture and might jeopardize the entire treatment cycle. Two large RCTs have shown that blastocyst culture after RIF following cleavage stage transfers resulted in significantly higher implantation and live birth rates. ${ }^{59,60}$ However, this approach should be adopted with caution considering the high embryonic aneuploidy rate in RIF cases, ${ }^{48,61}$ and the risk of loosing most or even all the embryos.

Use of the best transfer technique is mandatory in each cycle and obvious in RIF. Embryo transfer (ET) with soft atraumatic catheters under ultrasound guidance to assure midcavity placement is the superior and almost universally accepted standard in ART. ${ }^{62}$ Revision of the transfer history is mandatory in each RIF case in order to assure that no anatomical or other problem have precluded optimal smoothly performed ETs. In special cases, measures like cervical dilation or ET under general anesthesia are necessary to assure this goal. Many clinicians transfer large number of embryos after RIF. Even in countries in which the number of transferred embryos is limited by voluntary or mandatory regulations, the limit is lifted once RIFs occur. However, there is little evidence that the transfer of more than three embryos is beneficial in such case.

\section{Zona Hardening}

The zona pellucida (ZP) surrounding the oocyte hardens after fertilization, depolarization and spillage of the cortical granules, in order to prevent polyspermy and protect the integrity of the migrating embryo until its implantation. The appearance of a breach in the hard and nonadhesive ZP and blastocyst hatching is part of normal implantation. Increased ZP thickness and hardness was associated with lower implantation rates. ${ }^{63,64}$ Thus, failure of the ZP to rupture has been suggested as a possible cause of RIF, and different mechanical, chemical and optical techniques were used in order to regionally weaken the ZP or even create an opening in it in order to assist hatching ( $\mathrm{AH})$ and implantation. AH is not hazardous. A single report of an association between $\mathrm{AH}$ and monozygotic twinning was published but was not sustained by others. ${ }^{65} \mathrm{AH}$ was not found to improve the overall success rate of ART. ${ }^{66-68}$ However, evidence to the benefit of performing AH in selected RIF cases does exist. Cohen et al performed a randomized, prospective trial in which mechanical and chemical $\mathrm{AH}$ was found to be beneficial only if performed on embryos with a poor implantation potential (thick ZP, poor development, advanced maternal age) ${ }^{69}$ Obruca and associates used an Er:Yag laser system for AH in RIF cases resulting in increased implantation and pregnancy rates (14.4\% vs $6 \%$ and $40 \%$ vs $16.2 \%$ respectively). ${ }^{70}$ Chao, Magli and Nakayama et al have shown $\mathrm{AH}$, performed by different techniques, to increase pregnancy and implantation rates when selectively applied in patients with RIFs. ${ }^{71-73}$ The latter studies do support the notion that $\mathrm{AH}$, no matter how performed, might be beneficial in RIF cases. Despite the lack of uniformity in the study design and methods used, it seems that $\mathrm{AH}$ is beneficial in selected cases of poor prognosis, and bares no actual risk. We believe that $\mathrm{AH}$ by the method most familiar to the embryologist, should be performed in every RIF case.

\section{SUMMARY AND CONCLUSIONS}

RIF is a difficult unresolved challenge in reproductive medicine and a source of endless patient frustration and despair. Though far from resolution, several investigative measures and therapeutic interventions were found to be useful in this complex condition according to the published literature and our experience.

1. A repeated evaluation of the uterine cavity and eventual correction of any significant anomaly found should be performed in all cases.

2. An endometrial biopsy might be of both diagnostic and therapeutic value.

3. Hydrosalpinges should be surgically treated.

4. The benefit of thrombophilia screening and LMW heparin therapy is supported by some works and in our opinion should be a part of the initial evaluation.

5. All couples who experience RIF should have their karyotypes examined, and targeted PGD should be performed if a parental aberration is found. If the parental karyotypes are normal, the performance of PGS is of no benefit.

6. Couple HLA typing and cross-match are warranted in high order RIF if no other anomaly was detected. IVIG therapy should be offered if 3 or more HLA loci are common and the cross-match is negative.

7. The historical embryo culture reports should be investigated and condition changes should be considered. Blastocyst culture should be considered if embryos of adequate number and quality are available.

8. A procedure for assisted hatching should be performed in all RIF cases prior to embryo transfer.

\section{REFERENCES}

1. Tan BK, Vandekerckhove P, Kennedy R, Keay SD. Investigation and current management of recurrent IVF treatment failure in the UK. BJOG. Jun 2005;112(6):773-80.

2. Margalioth EJ, Ben-Chetrit A, Gal M, Eldar-Geva T. Investigation and treatment of repeated implantation failure following IVF-ET. Human Reproduction 21(12):3036-43. 
3. Demirol A, Gurgan T. Effect of treatment of intrauterine pathologies with office hysteroscopy in patients with recurrent IVF failure. Reprod Biomed Online May 2004;8(5):590-94.

4. Donnez J, Jadoul P. What are the implications of myomas on fertility? A need for a debate? Hum Reprod Jun 2002;17(6): 1424-30.

5. Bergh C, Hillensjo T, Nilsson L. Sonographic evaluation of the endometrium in in vitro fertilization IVF cycles. A way to predict pregnancy? Acta Obstet Gynecol Scand Dec 1992;71(8):62428.

6. Check JH, Nowroozi K, Choe J, Dietterich C. Influence of endometrial thickness and echo patterns on pregnancy rates during in vitro fertilization. Fertil Steril Dec 1991;56(6):117375.

7. Dickey RP, Olar TT, Curole DN, Taylor SN, Rye PH. Endometrial pattern and thickness associated with pregnancy outcome after assisted reproduction technologies. Hum Reprod Mar 1992;7(3):418-21.

8. Gonen Y, Casper RF, Jacobson W, Blankier J. Endometrial thickness and growth during ovarian stimulation: A possible predictor of implantation in in vitro fertilization. Fertil Steril Sep 1989;52(3):446-50.

9. Noyes N, Liu HC, Sultan K, Schattman G, Rosenwaks Z. Endometrial thickness appears to be a significant factor in embryo implantation in in vitro fertilization. Hum Reprod Apr 1995;10(4):919-22.

10. Sher G, Herbert C, Maassarani G, Jacobs MH. Assessment of the late proliferative phase endometrium by ultrasonography in patients undergoing in vitro fertilization and embryo transfer (IVF/ET). Hum Reprod Feb 1991;6(2):232-37.

11. Zenke U, Chetkowski RJ. Transfer and uterine factors are the major recipient-related determinants of success with donor eggs. Fertil Steril Oct 2004;82(4):850-56.

12. Hassan HA, Saleh HA. Endometrial unresponsiveness: A novel approach to assessment and prognosis in in vitro fertilization cycles. Fertil Steril Oct 1996;66(4):604-07.

13. De Geyter C, Schmitter M, De Geyter M, Nieschlag E, Holzgreve W, Schneider HP. Prospective evaluation of the ultrasound appearance of the endometrium in a cohort of 1,186 infertile women. Fertil Steril Jan 2000;73(1):106-13.

14. Fleischer AC, Herbert CM, Sacks GA, Wentz AC, Entman SS, James AE (Jr). Sonography of the endometrium during conception and nonconception cycles of in vitro fertilization and embryo transfer. Fertil Steril Sep 1986;46(3):442-47.

15. Glissant A, de Mouzon J, Frydman R. Ultrasound study of the endometrium during in vitro fertilization cycles. Fertil Steril Dec 1985;44(6):786-90.

16. Khalifa E, Brzyski RG, Oehninger S, Acosta AA, Muasher SJ. Sonographic appearance of the endometrium: The predictive value for the outcome of in vitro fertilization in stimulated cycles. Hum Reprod May 1992;7(5):677-80.

17. Oliveira JB, Baruffi RL, Mauri AL, Petersen CG, Campos MS, Franco JG (Jr). Endometrial ultrasonography as a predictor of pregnancy in an in vitro fertilization programme. Hum Reprod Aug 1993;8(8):1312-15.

18. Welker BG, Gembruch U, Diedrich K, al-Hasani S, Krebs D. Transvaginal sonography of the endometrium during ovum pickup in stimulated cycles for in vitro fertilization. J Ultrasound Med Oct 1989;8(10):549-53.

19. Rinaldi L, Lisi F, Floccari A, Lisi R, Pepe G, Fishel S. Endometrial thickness as a predictor of pregnancy after in vitro fertilization but not after intracytoplasmic sperm injection. Hum Reprod Jul 1996;11(7):1538-41.
20. Zhang X, Chen CH, Confino E, Barnes R, Milad M, Kazer RR. Increased endometrial thickness is associated with improved treatment outcome for selected patients undergoing in vitro fertilization-embryo transfer. Fertil Steril Feb 2005;83(2):33640.

21. Friedler S, Schenker JG, Herman A, Lewin A. The role of ultrasonography in the evaluation of endometrial receptivity following assisted reproductive treatments: A critical review. Hum Reprod Update Jul-Aug 1996;2(4):323-35.

22. Sundstrom P. Establishment of a successful pregnancy following in vitro fertilization with an endometrial thickness of no more than 4 mm. Hum Reprod Jun 1998;13(6):1550-52.

23. Weckstein LN, Jacobson A, Galen D, Hampton K, Hammel J. Low-dose aspirin for oocyte donation recipients with a thin endometrium: Prospective, randomized study. Fertil Steril Nov 1997;68(5):927-30.

24. Sher G, Fisch JD. Effect of vaginal sildenafil on the outcome of in vitro fertilization (IVF) after multiple IVF failures attributed to poor endometrial development. Fertil Steril Nov 2002;78(5): 1073-76.

25. Tourgeman DE, Slater CC, Stanczyk FZ, Paulson RJ. Endocrine and clinical effects of micronized estradiol administered vaginally or orally. Fertil Steril Jan 2001;75(1):200-02.

26. Gelbaya TA, Kyrgiou M, Li TC, Stern C, Nardo LG. Low-dose aspirin for in vitro fertilization: A systematic review and metaanalysis. Hum Reprod Update Jul-Aug 2007;13(4):357-64.

27. Shufaro Y, Simon A, Laufer N, Fatum M. Thin unresponsive endometrium: A possible complication of surgical curettage compromising ART outcome. J Assist Reprod Genet Aug 2008;25(8):421-25.

28. Barash A, Dekel N, Fieldust S, Segal I, Schechtman E, Granot I. Local injury to the endometrium doubles the incidence of successful pregnancies in patients undergoing in vitro fertilization. Fertil Steril June 2003;79(6):1317-22.

29. Raziel A, Schachter M, Strassburger D, Bern O, Ron-El R, Friedler S. Favorable influence of local injury to the endometrium in intracytoplasmic sperm injection patients with highorder implantation failure. Fertil Steril Jan 2007;87(1):198-201.

30. The influence of hydrosalpinx on IVF and embryo transfer: A review. Hum Reprod Update Jul-Aug 2000;6(4):387-95.

31. Katz E, Akman MA, Damewood MD, Garcia JE. Deleterious effect of the presence of hydrosalpinx on implantation and pregnancy rates with in vitro fertilization. Fertil Steril Jul 1996;66(1):122-25.

32. Johnson N, van Voorst S, Sowter MC, Strandell A, Mol BW. Surgical treatment for tubal disease in women due to undergo in vitro fertilisation. Cochrane Database Syst Rev (1): CD002125.

33. Salpingectomy for hydrosalpinx prior to in vitro fertilization. Fertil Steril Nov 2008;90(5 Suppl):S66-68.

34. Ledee-Bataille N, Bonnet-Chea K, Hosny G, Dubanchet S, Frydman R, Chaouat G. Role of the endometrial tripod interleukin-18, -15, and -12 in inadequate uterine receptivity in patients with a history of repeated in vitro fertilization-embryo transfer failure. Fertil Steril Mar 2005;83(3):598-605.

35. Inagaki N, Stern C, Mc Bain J, Lopata A, Kornman L, Wilkinson D. Analysis of intrauterine cytokine concentration and matrix-metalloproteinase activity in women with recurrent failed embryo transfer. Hum Reprod Mar 2003;18(3):608-15.

36. Brosens J, Verhoeven H, Campo R, Gianaroli L, Gordts S, Hazekamp J, et al. High endometrial aromatase P450 mRNA expression is associated with poor IVF outcome. Hum Reprod Feb 2004;19(2):352-56. 
37. Pantos K, Nikas G, Makrakis E, Stavrou D, Karantzis P, Grammatis M. Clinical value of endometrial pinopodes detection in artificial donation cycles. Reprod Biomed Online July 2004;9(1):86-90

38. Denis AL, Guido M, Adler RD, Bergh PA, Brenner C, Scott RT (Jr). Antiphospholipid antibodies and pregnancy rates and outcome in in vitro fertilization patients. Fertil Steril June 1997;67(6):1084-90.

39. Eldar-Geva T, Wood C, Lolatgis N, Rombauts L, Kovacs G, Fuscaldo J, et al. Cumulative pregnancy and live birth rates in women with antiphospholipid antibodies undergoing assisted reproduction. Hum Reprod June 1999;14(6):146166.

40. Azem F, Many A, Yovel I, Amit A, Lessing JB, Kupferminc MJ. Increased rates of thrombophilia in women with repeated IVF failures. Human Reproduction February 1, 2004;19(2): 368-70.

41. Qublan HS, Eid SS, Ababneh HA, Amarin ZO, Smadi AZ, Al-Khafaji FF, et al. Acquired and inherited thrombophilia: Implication in recurrent IVF and embryo transfer failure. Human Reproduction 21(10):2694-98.

42. Qublan H, Amarin Z, Dabbas M, Farraj AE, Beni-Merei Z, AlAkash $\mathrm{H}$, et al. Low-molecular-weight heparin in the treatment of recurrent IV/ET failure and thrombophilia: A prospective randomized placebo-controlled trial. Human Reproduction 2008; 11(4):246-53.

43. Bellver J, Soares SR, Ãlvarez C, Muñoz E, RamÃ-rez A, Rubio C, et al. The role of thrombophilia and thyroid autoimmunity in unexplained infertility, implantation failure and recurrent spontaneous abortion. Human Reproduction February 1, 2008;23(2):278-84.

44. Martinelli I, Battaglioli T, Mannucci PM. Screening of thrombophilia in women with failure of embryo implantation: Far from being recommended. Haematologica Dec 2003; 88(12): ELT36.

45. Martinelli I, Taioli E, Ragni G, Levi-Setti P, Passamonti SM, Battaglioli T, et al. Embryo implantation after assisted reproductive procedures and maternal thrombophilia. Haematologica July 2003;88(7):789-93.

46. Carp HJ, Toder V, Mashiach S, Rabinovici J. Effect of paternal leukocyte immunization on implantation after biochemical pregnancies and repeated failure of embryo transfer. Am J Reprod Immunol Mar-Apr 1994;31(2-3):112-15.

47. Elram T, Simon A, Israel S, Revel A, Shveiky D, Laufer N. Treatment of recurrent IVF failure and human leukocyte antigen similarity by intravenous immunoglobulin. Reprod Biomed Online Dec 2005;11(6):745-49.

48. Pehlivan T, Rubio C, Rodrigo L, Romero J, Remohi J, Simon C, et al. Impact of preimplantation genetic diagnosis on IVF outcome in implantation failure patients. Reprod Biomed Online Mar 2003;6(2):232-37.

49. Voullaire L, Wilton L, McBain J, Callaghan T, Williamson R. Chromosome abnormalities identified by comparative genomic hybridization in embryos from women with repeated implantation failure. Mol Hum Reprod Nov 2002;8(11): 1035-41.

50. Rubio C, Gil-Salom M, Simon C, Vidal F, Rodrigo L, Minguez $Y$, et al. Incidence of sperm chromosomal abnormalities in a risk population: Relationship with sperm quality and ICSI outcome. Hum Reprod Oct 2001;16(10):2084-92.

51. Tarlatzis BC, Toncheva DI, Vatev IT. Significance of chromosomal aberrations for the unsuccessful procedures of assisted reproduction. Eur J Obstet Gynecol Reprod Biol Feb 2000;88(2):181-87.

52. Raziel A, Friedler S, Schachter M, Kasterstein E, Strassburger D, Ron-El R. Increased frequency of female partner chromosomal abnormalities in patients with high-order implantation failure after in vitro fertilization. Fertil Steril Sep 2002;78(3):515-19.

53. Munne S, Sandalinas M, Escudero T, Velilla E, Walmsley R, Sadowy S, et al. Improved implantation after preimplantation genetic diagnosis of aneuploidy. Reprod Biomed Online JulAug 2003;7(1):91-97.

54. Wilding M, Forman R, Hogewind G, Di Matteo L, Zullo F, Cappiello F, et al. Preimplantation genetic diagnosis for the treatment of failed in vitro fertilization-embryo transfer and habitual abortion. Fertil Steril May 2004;81(5):1302-07.

55. Mastenbroek S, Twisk M, van Echten-Arends J, SikkemaRaddatz B, Korevaar JC, Verhoeve HR, et al. In Vitro fertilization with preimplantation genetic screening. New England Journal of Medicine 2007;357(1):9-17.

56. Jayot S, Parneix I, Verdaguer S, Discamps G, Audebert A, Emperaire JC. Coculture of embryos on homologous endometrial cells in patients with repeated failures of implantation. Fertil Steril Jan 1995;63(1):109-14.

57. Simon C, Mercader A, Garcia-Velasco J, Nikas G, Moreno C, Remohi J, et al. Coculture of human embryos with autologous human endometrial epithelial cells in patients with implantation failure. J Clin Endocrinol Metab Aug 1999;84(8):2638-46.

58. Spandorfer SD, Pascal P, Parks J, Clark R, Veeck L, Davis OK, et al. Autologous endometrial coculture in patients with IVF failure: Outcome of the first 1,030 cases. J Reprod Med June 2004;49(6):463-67.

59. Guerif F, Bidault R, Gasnier O, Couet ML, Gervereau O, Lansac J, et al. Efficacy of blastocyst transfer after implantation failure. Reprod Biomed Online Dec 2004;9(6):630-36.

60. Levitas E, Lunenfeld E, Har-Vardi I, Albotiano S, Sonin Y, Hackmon-Ram R, et al. Blastocyst-stage embryo transfer in patients who failed to conceive in three or more day 2-3 embryo transfer cycles: A prospective, randomized study. Fertil Steril Mar 2004;81(3):567-71.

61. Voullaire L, Wilton L, McBain J, Callaghan T, Williamson R. Chromosome abnormalities identified by comparative genomic hybridization in embryos from women with repeated implantation failure. Molecular Human Reproduction November 1, 2002;8(11):1035-41.

62. Sallam HN. Embryo transfer: Factors involved in optimizing the success. Curr Opin Obstet Gynecol June 2005;17(3): 289-98.

63. Cohen J, Malter H, Wright G, Kort H, Massey J, Mitchell D. Partial zona dissection of human oocytes when failure of zona pellucida penetration is anticipated. Hum Reprod May 1989;4(4):435-42.

64. De Vos A, Van Steirteghem A. Zona hardening, zona drilling and assisted hatching: New achievements in assisted reproduction. Cells Tissues Organs 2000;166(2):220-27.

65. Hershlag A, Paine T, Cooper GW, Scholl GM, Rawlinson K, Kvapil G. Monozygotic twinning associated with mechanical assisted hatching. Fertil Steril Jan 1999;71(1):144-46.

66. Hellebaut S, De Sutter P, Dozortsev D, Onghena A, Qian C, Dhont $\mathrm{M}$. Does assisted hatching improve implantation rates after in vitro fertilization or intracytoplasmic sperm injection in all patients? A prospective randomized study. J Assist Reprod Genet Jan 1996;13(1):19-22. 
67. Hurst BS, Tucker KE, Awoniyi CA, Schlaff WD. Assisted hatching does not enhance IVF success in good-prognosis patients. J Assist Reprod Genet Feb 1998;15(2):62-64.

68. Lanzendorf SE, Nehchiri F, Mayer JF, Oehninger S, Muasher SJ. A prospective, randomized, double-blind study for the evaluation of assisted hatching in patients with advanced maternal age. Hum Reprod Feb 1998;13(2):409-13.

69. Cohen J, Alikani M, Trowbridge J, Rosenwaks Z. Implantation enhancement by selective assisted hatching using zona drilling of human embryos with poor prognosis. Hum Reprod May 1992;7(5):685-91.

70. Obruca A, Strohmer H, Sakkas D, Menezo Y, Kogosowski A, Barak Y, et al. Use of lasers in assisted fertilization and hatching. Hum Reprod Sep 1994;9(9):1723-26.
71. Magli MC, Gianaroli L, Ferraretti AP, Fortini D, Aicardi G, Montanaro N. Rescue of implantation potential in embryos with poor prognosis by assisted zona hatching. Hum Reprod May 1998;13(5):1331-35.

72. Nakayama T, Fujiwara H, Yamada S, Tastumi K, Honda T, Fujii S. Clinical application of a new assisted hatching method using a piezo-micromanipulator for morphologically low-quality embryos in poor-prognosis infertile patients. Fertil Steril June 1999;71(6):1014-18.

73. Chao KH, Chen SU, Chen HF, Wu MY, Yang YS, Ho HN. Assisted hatching increases the implantation and pregnancy rate of in vitro fertilization (IVF)-embryo transfer (ET), but not that of IVF-tubal ET in patients with repeated IVF failures. Fertil Steril May 1997;67(5):904-08. 